\title{
Wirkungen von Standortverlagerung und Ausgliederung auf die Arbeitsbedingungen
}

Nokia und die Deutsche Telekom sind keine Einzelfälle: Viele Unternehmen in Deutschland verlagern ihre Standorte oder gliedern Funktionsbereiche aus; auch mittlere und kleine Betriebe. Welche Dimensionen nehmen Standortverlagerungen und Ausgliederungen in Deutschland an? Umstrukturierungsmaßnahmen verändern die Betriebe weit über den gefürchteten Arbeitsplatzabbau hinaus. Welche Auswirkungen hat dies auf die Beschäftigten und die Arbeitsbedingungen?

\section{Vorbemerkung}

In den letzten Jahren ist der Druck zur Veränderung in weiten Teilen der Wirtschaft gestiegen und hat in den Unternehmen u.a. tiefgreifende Umstrukturierungsaktivitäten ausgelöst. Unternehmen haben aufgrund veränderter Wettbewerbsbedingungen Produktionen und Betriebsstätten verlagert, betriebliche Funktionen wie Buchhaltung, EDV etc. ausgegliedert und rechtlich verselbstständigt, sich auf Kerngeschäfte konzentriert, den Unternehmenswert gesteigert, indem sie trotz guter Gewinnlage Lohnkürzungen und Arbeitsplatzabbau durchsetzten. Zusätzlich erhöhen sie vielerorts durch Fusionen und Zukäufe die eigene Marktmacht.

Umstrukturierung bedeutet „bestehende Strukturen zu verändern bzw. sie neu zu gestalten" (Hofmann et al., S. 7). Auf Ebene des Betriebs kann es sich dabei um Veränderungen hinsichtlich des rechtlichen Status, der Eigentumsverhältnisse, des betrieblichen Aufbaus oder einzelner Prozessabläufe handeln. In der Regel setzt sich eine Umstrukturierung aus mehreren Einzelmaßnahmen zusammen. Im Fokus des vorliegenden Beitrags stehen die Standortverlagerung (Offshoring) sowie die Ausgliederung (Outsourcing). Beides sind Umstrukturierungsmaßnahmen mit erheblichen Auswirkungen auf die Beschäftigten.

Eine einheitliche Begrifflichkeit von Standortverlagerung und Outsourcing existiert bislang nicht. Die ursprüngliche Bedeutung umfasst Maßnahmen eines Unternehmens zur vollständigen oder teilweisen Umsiedlung und Umwandlung von Betrieben und Betriebsteilen. Dies kann komplette Betriebsstätten, einzelne Funktions- bereiche oder auch nur bestimmte Arbeitsschritte betreffen, die räumlich oder rechtlich ausgelagert werden. Auslagerungen können ins Umland oder Ausland erfolgen, stets aber verbleibt der betroffene Teil im engen wirtschaftlichen Verbund des Unternehmens. Wir verstehen unter Standortverlagerung (Dressler 2007), wenn ein Betrieb oder ein Betriebsteil in eine Niederlassung im Inland oder Ausland verlagert wird. Dabei bleibt die Niederlassung Teil des rechtlichen Unternehmens- bzw. Konzernverbundes. Demgegenüber verstehen wir unter Ausgliederung die Abgabe von Unternehmensaufgaben und -strukturen an Fremdfirmen, auch wenn diese vom Ursprungsunternehmen gegründet wurden. Ein zeitlich befristeter Vertrag verpflichtet Letztere zur Erbringung ausgewählter Produktionen oder Dienstleistungen. Hierzulande oft nur mit Auslagerungsaktivitäten innerhalb der Bundesrepublik verknüpft, wird dieser Begriff in der Managementliteratur längst auch für grenzüberschreitende Auslagerungsaktivitäten benutzt (International Assocation of Outsourcing Professionals; www.outsourcingprofessional.org).

Bereits in der letzten WSI-Betriebsrätebefragung 2004/2005 wurden Betriebsräte nach ihren Erfahrungen mit Standortverlagerungen befragt. In der WSI-Betriebsrätebefragung 2007 wurde diese Befragung wiederholt und um das Thema Ausgliederung erweitert. Darüber erhielten wir einerseits Informationen zum Standort- und Ausgliederungsgeschehen in deutschen Betrieben aus Sicht der Betriebsräte und andererseits ihre Einschätzung, wie sich diese beiden Umstrukturierungsformen auf die Beschäftigten und ihre Arbeitsbedingungen ausgewirkt haben.

Jede Umstrukturierung hat Auswirkungen auf Arbeitsplätze und Beschäftigte, da sie sich doch nicht selten als effektive Rationalisierungsmethode darstellt, mit de- ren Hilfe Unternehmen Kosten einsparen wollen. Die Ergebnisse der letzten Befragung zeigten dies deutlich (Ahlers et al. 2007). Alleine schon die betriebliche Diskussion um Standortverlagerung übte häufig Druck auf die Beschäftigten aus, die Einschnitte bei den Arbeitsbedingungen, bei der Arbeitszeit oder auch bei der Entlohnung hinnehmen mussten und trotzdem immer in der Sorge waren, dass in beschäftigungspolitisch schwierigen Zeiten für ihre im Zusammenhang mit einer Standortverlagerung möglicherweise auch wegfallenden Arbeitsplätze nicht genug alternative Beschäftigungsmöglichkeiten entstanden.

In Deutschland beschäftigt sich die Forschung systematisch mit dem Wandel von Unternehmensstrategien und der Entwicklung der deutschen Wirtschaft im globalen Kontext. Es überrascht nicht, dass sich die Diskussionen vornehmlich auf die Folgen und Anforderungen für Volkswirtschaften und Unternehmen beziehen. Die Beschäftigten kommen darin oft nur negativ als Kostenfaktor oder im Hinblick auf vorhandene Qualifikationen als positiver „Standortaspekt" vor. Insgesamt nehmen die Konsequenzen für Entlohnung, Arbeitsverhältnisse und Arbeitsbedingungen in der Forschung nur einen bescheidenen Raum ein (Haipeter 2007). Im Ergebnis ist deshalb der wissenschaftliche Kenntnisstand über die Konsequenzen von verschiedenen Umstrukturierungsmaßnahmen für Beschäftigte bislang gering. Und dies, obwohl die Vermutung naheliegt, dass für die Belegschaften in den Betrieben, in

Astrid Ziegler, Dr., ist Wissenschaftlerin im WSI in der Hans-Böckler-Stiftung. Arbeitsschwerpunkt: Strukturforschung und -politik. e-mail: Astrid-Zieg/er@boeckler.de 


\begin{tabular}{|c|c|c|}
\hline $\begin{array}{l}\text { Anteil der Betriebe, deren Betriebsräte } \\
\text { sich mit dem Thema ... }\end{array}$ & $\begin{array}{c}\text { Standort- } \\
\text { verlagerung }\end{array}$ & $\begin{array}{l}\text { Aus- } \\
\text { gliederung }\end{array}$ \\
\hline befassen mussten & 16,4 & 23,4 \\
\hline \multicolumn{3}{|l|}{ davon der Anteil der Betriebe ${ }^{*}$} \\
\hline die aktuell diskutieren & 24,6 & 24,7 \\
\hline die aktuell verlagern/ausgliedern & 22,9 & 17,7 \\
\hline die nach 2005 verlagerten/ausgliederten & 46,8 & 48,9 \\
\hline die vor 2005 verlagerten/ausgliederten & 67,1 & 64,2 \\
\hline
\end{tabular}

denen Ausgliederungen und Verlagerungen angekündigt werden, wie in Betrieben, die sich um die Übernahme entsprechender Aufgaben und Funktionsbereiche bemühen, die Unsicherheit wächst. Und damit wird - hier wie dort - der Druck vonseiten der Geschäftsführung auf die Beschäftigten bzw. auf die betrieblichen Interessenvertretungen größer, Konzessionen einzugehen.

Der vorliegende Beitrag setzt an diesem Punkt an: Im ersten Schritt wird das Ausmaß von Standortverlagerung und Ausgliederung anhand der WSI-Betriebsrätebefragung 2007 analysiert (Abschnitt 2) und ihre Einflussgrößen untersucht (Abschnitt 3 und 4). Im zweiten Schritt steht die Frage nach den Auswirkungen auf Arbeitsplatzentwicklungen und Arbeitsbedingungen von Offshoring und Ausgliederung im Mittelpunkt (Abschnitt 5). Der Beitrag mündet in ein Fazit (Abschnitt 6), in dem die rückblickende Einschätzung von Umstrukturierungsmaßnahmen und der Bedarf an tariflicher Regulierung thematisiert wird.

\section{Ausmaß von Standort- verlagerung und Ausgliederung}

Über Standortverlagerung und Ausgliederung wird in der breiten Öffentlichkeit seit etlichen Jahren immer dann viel und kontrovers diskutiert, wenn - wie in jüngster Vergangenheit die Beispiele Nokia, AEG, BenQ, Karmann oder Deutsche Telekom zeigen - Großunternehmen und eine große Anzahl von Arbeitsplätzen betroffen sind. Aus solchen öffentlichkeitswirksamen Fällen wird dann gewöhnlich eine neue Quantität und auch Qualität des Verlagerungsund Ausgliederungsgeschehens in Deutsch- land abgeleitet. Tatsächlich sind Standortverlagerungen und Ausgliederungen keine ausschließlich großbetrieblichen Phänomene mehr, denn mittlerweile sind diese auch für kleine und mittlere Unternehmen eine Option geworden. Es sind außerdem nicht allein Unternehmen in Deutschland oder in den anderen westeuropäischen Staaten, die ausgliedern und/oder verlagern; auch Unternehmen in den bisherigen Zielländern (wie z. B. die osteuropäischen EU-Mitgliedsländer oder die asiatischen Schwellenländer) nutzen diese betrieblichen Möglichkeiten. Gleichzeitig werden nicht mehr nur einfache, arbeitsintensive Produktionen verlagert bzw. ausgegliedert; aufgrund moderner Informations- und Kommunikationstechnologien und geringer Transportkosten sind auch anspruchsvolle Fertigungen und hochwertige Dienstleistungen (wie z. B. Forschung und Entwicklung) betroffen.

In den letzten Jahren gab es in fast jedem zweiten Betrieb mit mehr als 20 Beschäftigten und einem Betriebsrat betriebliche Umstrukturierungsmaßnahmen. ${ }^{1}$ Nach der WSI-Betriebsrätebefragung 2007 gehören Standortverlagerung und Outsourcing mittlerweile zum betrieblichen Alltag. In $16,4 \%$ aller vom WSI befragten Betriebe mit Betriebsräten spielten Standortverlagerungen eine Rolle. Noch häufiger mussten sich Betriebsräte mit dem Thema Ausgliederung beschäftigen; fast jeder vierte befragte Betriebsrat $(23,4 \%)$ gab an, dass Outsourcing im Betrieb umgesetzt wurde (Tabelle 1). Damit haben Ausgliederungsprozesse quantitativ in deutschen Betrieben eine höhere Bedeutung als Standortverlagerungen.

Nach unseren Ergebnissen sind Standortverlagerungen und Ausgliederungen keine kurzfristigen Phänomene. Das Gros der Standortverlagerungen und Ausgliederungen fand vor 2005 statt und betraf rund zwei Drittel aller Unternehmen. Dabei wa- ren die Betriebsräte in der Vergangenheit etwas häufiger mit dem Thema Verlagerung beschäftigt als mit Outsourcing. Aber auch seit 2005 wurde in fast jedem zweiten betroffenen Betrieb verlagert bzw. ausgegliedert. In 13,7 \% der verlagernden und ausgliedernden Betriebe wurden seit 2005 sogar beide Umstrukturierungsformen genutzt. Betriebe verlagern bzw. gliedern Funktionsbereiche nicht nur einmal aus. Seit 2005 erfolgten in den betroffenen Betrieben durchschnittlich 2,3 Standortverlagerungen und 2,8 Ausgliederungen. Auch aktuell wird nicht nur über die Möglichkeiten von Standortverlagerung und Ausgliederung diskutiert, sondern in fast $18 \%$ der Betriebe wird derzeit ausgegliedert und sogar in $23 \%$ verlagert.

\section{Motive der Standort- verlagerung und Ausgliederung}

$\mathrm{Ob}$ nun eine Standortverlagerung oder Ausgliederung aus beschäftigungspolitischer Sicht negativ oder positiv zu beurteilen ist, hängt auch von den konkreten Motiven ab, die zu der Maßnahme führen. Entscheidend für eine unternehmerische Standortpolitik sind mehrere Faktoren wie Fertigungskosten, Qualifikation, Vorhandensein von Infrastruktur sowie Markterschließung oder Kundennähe, die in die unternehmerische Entscheidung für oder gegen einen Standort bzw. eine Umstrukturierungsmaßnahme einfließen. Die Frage nach den Motiven der Standortverlagerung oder der Ausgliederung ist daher wichtig, wenn man ihre Auswirkungen auf die Beschäftigten im Blick hat. Die entsprechenden Argumentationsmuster lauten wie folgt:

\section{(1) Standortverlagerung}

In der Literatur finden sich zwei theoretische Erklärungsansätze für Standortverlagerungen von Unternehmen. ${ }^{2}$ Bei Verlagerungen zur Einsparung von Produktions-

\footnotetext{
Die Autorin dankt Wolfram Brehmer für wertvolle Hilfe und Unterstützung bei der Datenanalyse, ohne die der vorliegende Beitrag nicht zustande gekommen wäre.

2 Zu den Motiven im Automobilsektor vgl. die aktuel le Untersuchung von Kinkel/Spomenka (2008).
} 
kosten dürfte der Aufbau einer Produktionsstätte an kostengünstigen Standorten negative Auswirkungen auf die Arbeitnehmer am bisherigen Standort des Unternehmens haben. Für die Beschäftigten können sich die Arbeitsbedingungen verschlechtern oder im schlimmsten Fall zum Arbeitsplatzverlust führen, weil der alte Standort aus Kostengründen (teilweise) aufgegeben wird. Problematisch wird es dann für die Betroffenen, wenn der regionale Arbeitsmarkt keine alternative Beschäftigungsmöglichkeit bietet und sie in die Arbeitslosigkeit entlassen werden. Bei Standortverlagerungen als Mittel zur Markterschließung, wenn Unternehmen z. B. über ihr ausländisches Engagement in erster Linie neue Märkte erschließen wollen und ergänzend zum Heimatstandort Produktion oder (Vertriebs-)Strukturen im Ausland aufbauen, sichert das so generierte Umsatzwachstum die heimischen Arbeitsplätze. Dies führt im günstigsten Fall sogar zum Stellenaufbau am deutschen Standort.

\section{(2) Ausgliederung}

Ausgliederungsaktivitäten werden vorwiegend unter Kostengesichtspunkten diskutiert. Die Ausgliederung von Vertriebs- oder Serviceleistungen und deren anschließender Ankauf sei für das Unternehmen billiger - so die Argumentation. Weitere Motive, die im Zusammenhang mit dem Thema Ausgliederung diskutiert werden, sind die Reorganisation des Betriebes und die Konzentration auf seine Kernkompetenzen. In der Regel gehen die Arbeitsplätze vom Stammunternehmen in den ausgegliederten Betrieb über - mit oftmals schlechteren Bedingungen für die Beschäftigten.

Während also die Anpassung des Unternehmens an den veränderten wirtschaftlichen Kontext (u. a. Märkte, Zulieferer, Kunden) für Standortverlagerung das auslösende Moment sein kann, wird die andere Seite der Medaille bei beiden Umstrukturierungsmaßnahmen die Senkung der Kosten und die Steigerung des Gewinns sein. Vor allem im zweiten Fall kann die Belegschaft erheblich unter Druck geraten. Mitgestaltungsmöglichkeiten wie auch Verhandlungsspielräume dürften dabei wegen der „Kraft des Faktischen“ eher beschränkt sein.

Nach Angaben der Betriebsräte ist bei beiden Umstrukturierungsmaßnahmen der wichtigste Grund die Reduktion der Personalkosten. Für $58 \%$ der Betriebsräte mit

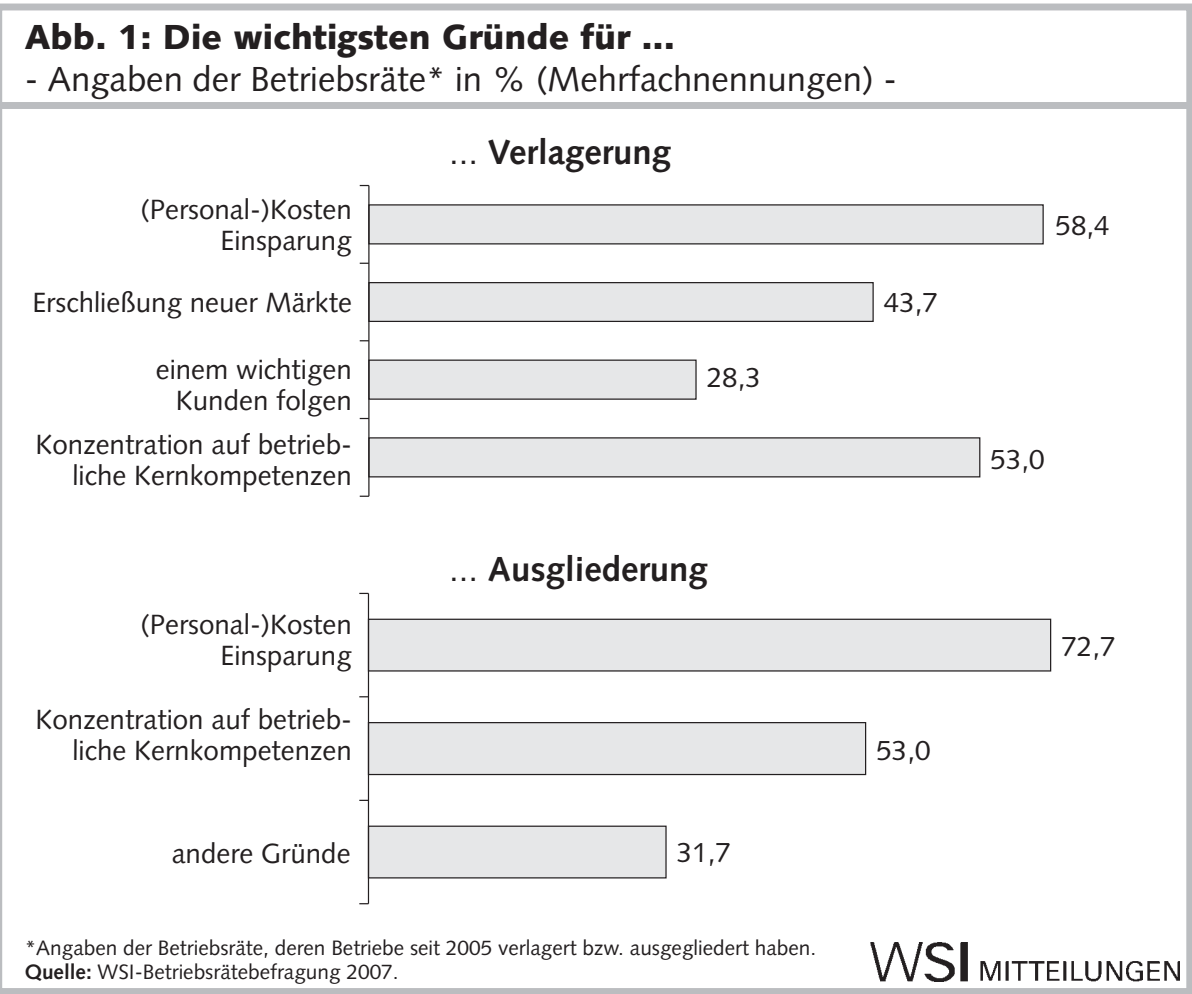

Verlagerungserfahrung und sogar fast $73 \%$ der Betriebsräte mit Ausgliederungserfahrung war dies der Hauptgrund, der ihnen von der Unternehmensleitung für die Verlagerungs- und Ausgliederungsentscheidung des Betriebes genannt wurde. Mehr als jeder zweite Betrieb verlagerte bzw. gliederte gleichzeitig aus, um sich auf betriebliche Kernkompetenzen zu konzentrieren. Bei den Verlagerungsbetrieben spielte zusätzlich die Erschließung neuer Märkte im Ausland eine Rolle. Ein Teil der Betriebe folgte zudem einem wichtigen Kunden. Dadurch soll eine größere Kundennähe erreicht werden, die über den reinen Export nicht $\mathrm{zu}$ realisieren wäre. Diese Befragungsergebnisse über die Beweggründe für Offshoring und Outsourcing decken sich zumindest bei der Verlagerung mit den gängigen Untersuchungen der letzten Zeit (Kinkel et al. 2005; DIHK 2005), wonach der Kostenaspekt nach wie vor dominiert.

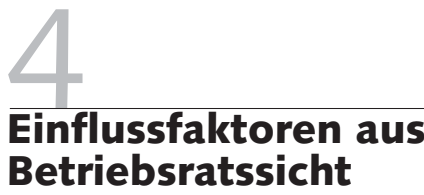

Was zeichnet aus Sicht der Betriebsräte die Betriebe aus, die seit 2005 verlagert oder ausgegliedert haben? Wie unterscheiden diese sich von den Betrieben, die diese Umstrukturierungsmaßnahmen nicht genutzt haben im Hinblick auf beschäftigungsrelevante Aspekte?

Diese Fragen werden anhand von zwei logistischen Regressionen mit den Daten aus der WSI-Betriebsrätebefragung 2007 beantwortet. Anders als die gängigen $\mathrm{Da}-$ tenquellen zu Umstrukturierungen bietet die WSI-Betriebsrätebefragung 2007 aus Sicht der betrieblichen Interessenvertretungen Informationen zu innerbetrieblichen Strukturveränderungen, zu Veränderungen der Arbeitsbedingungen, Arbeitsweisen und zum Verhältnis von Betriebsrat und Arbeitgeber. Keine bzw. lediglich grobe Informationen liefert sie dagegen zu der wirtschaftlichen Situation der Betriebe bzw. zur Einbindung der Betriebe in internationale Wertschöpfungsketten. Üblicherweise werden die klassischen Kriterien wie Umsatz, Gewinn, Exportquote etc. in den Analysen zu Offshoring und Outsourcing herangezogen (Görzig et al. 2005; Buch/ Schnitzer 2007). Bei der WSI-Betriebsrätebefragung fließen demgegenüber andere Kennzahlen in die Analyse ein, die sonst normalerweise nicht berücksichtigt werden. Sie ist - wie keine andere Datenquelle - dafür geeignet, die Auswirkungen von Standortverlagerung und Ausgliederung auf die Beschäftigten und ihre Arbeitsbedingungen zu quantifizieren. Die in der Analyse berücksichtigten Daten decken annähernd die Bereiche tarifliche Regelungen, Mitwirkungsspielraum des Betriebs- 
Tabelle 2: Einfluss- und Kontextfaktoren für Standortverlagerung und Ausgliederung

\begin{tabular}{|c|c|c|}
\hline Unabhängige Variablen & $\begin{array}{c}\text { Standortverlagerung } \\
\text { Odds-Ratio }\end{array}$ & $\begin{array}{c}\text { Ausgliederung } \\
\text { Odds-Ratio }\end{array}$ \\
\hline \multicolumn{3}{|l|}{ Allgemeine betriebliche Angaben } \\
\hline Ausgliederung bzw. Standortverlagerung seit 2005 & $1,99 * * *$ & $2,01 * * *$ \\
\hline Ostdeutschland & 0,94 & 1,1 \\
\hline \multicolumn{3}{|l|}{ Wirtschaftsbereiche } \\
\hline Grundstoff- und Produktionsgüterindustrie & $2,31 * * *$ & 0,78 \\
\hline Investitions- und Gebrauchsgüterindustrie & $2,05 * * *$ & 0,88 \\
\hline Verbrauchsgüterindustrie & 0,84 & 1,38 \\
\hline Baugewerbe & $1,89 *$ & 0,67 \\
\hline Handel (und Reparatur) & 1,48 & 0,88 \\
\hline Verkehr und Nachrichten & 1,49 & 1,48 \\
\hline Kredit und Versicherungen & $2,58 * * *$ & $1,72 *$ \\
\hline Sonstige Branchen & 0,68 & 1,21 \\
\hline \multicolumn{3}{|l|}{ (Referenz: private und öffentliche Dienstleistungen) } \\
\hline \multicolumn{3}{|l|}{ Beschäftigte und Entlohnung } \\
\hline Gesamtbeschäftigte & $1,00 * *$ & $1,00 * * *$ \\
\hline Leiharbeitnehmer vorhanden & $1,79 * * *$ & $1,79 * * *$ \\
\hline Personalabbau in den letzten zwei Jahren & $1,57 * *$ & $2,23 * * *$ \\
\hline Massenentlassungen in den letzten 10 Jahren & 0,91 & $1,42 * * *$ \\
\hline Bezahlte Überstunden & 1,13 & $1,39 * *$ \\
\hline Unbezahlte Überstunden & $1,43 * *$ & 0,94 \\
\hline \multicolumn{3}{|l|}{ Tarif und Mitbestimmung } \\
\hline Haustarifvertrag & 0,87 & $1,43 * *$ \\
\hline Branchentarifvertrag & 0,96 & 0,96 \\
\hline Häufig oder manchmal Mitwirkungsrechte behindert & 0,99 & $1,71 * * *$ \\
\hline Tarifpolitische Regelung zur betrieblichen Umstrukturierung & 1,13 & $1,58 * * *$ \\
\hline \multicolumn{3}{|l|}{ Betriebliche Probleme } \\
\hline Personalabbau/Beschäftigungssicherung & $2,53 * * *$ & 1,29 \\
\hline Sozialplan/Interessenausgleich & $4,84 * * *$ & 1,19 \\
\hline Ausgliederung/Schließung/Zusammenlegung & $4,49 * * *$ & $3,85^{* * *}$ \\
\hline Einschränkung betrieblicher Sozialleistungen & $3,83 * * *$ & 1,07 \\
\hline Pseudo $\mathrm{R}^{2}$ McFadden & 0,16 & 0,15 \\
\hline Anzahl der Fälle & $2.053^{* * *}$ & $2.053^{* * *}$ \\
\hline
\end{tabular}

rates und betriebliche Probleme ab. Über diese Informationen erhält man Hinweise, ob und wie sich Unternehmen, die verlagert oder ausgegliedert haben, von den anderen Betrieben unterscheiden. Wenn wie aus der Diskussion über die Gründe vermutet - Standortverlagerung und Ausgliederung erhebliche Auswirkungen auf die Beschäftigten und auf das Arbeitsklima haben, dürften diese Variablen einen signifikanten Einfluss zeigen.

In Tabelle 2 sind alle in die Schätzungen eingegangenen Einflussfaktoren zusammengetragen. Mit den Variablen wurden zwei separate Schätzungen zu den betrieblichen Einflussfaktoren durchgeführt, die Standortverlagerung bzw. Ausgliederung bedingen. Die Ergebnisse geben Auskunft über die Struktur der betroffenen Betriebe aus Sicht von Betriebsräten. Ziel ist es auch, Unterschiede zwischen den Betrieben hinsichtlich der beiden Umstrukturierungsmaßnahmen herauszufinden. Aus diesem

den innerbetrieblichen Arbeitsmarkt. Betriebe nutzten ihre Handlungsspielräume nicht nur für Umstrukturierung, sondern auch, um die Belegschaft flexibel und ihren Marktbedingungen entsprechend einzusetzen, insbesondere wenn Ausgliederung im Spiel war. Der Einsatz von Leiharbeitern im Betrieb sowie bezahlte und unbezahlte Überstunden waren nur zwei Aspekte eines flexiblen Arbeitsmarktes, die einen signifikant positiven Einfluss auf
Standortverlagerung und Ausgliederung ausübten.

Andere Studien weisen für das verarbeitende Gewerbe nach (u. a. Kinkel et al. 2004), dass mit steigender Betriebsgröße der Anteil der Betriebe mit Produktionsverlagerungen anstieg. Untersuchungen zu jüngeren Verlagerungsaktivitäten schließen kleine und mittlere Unternehmen mit ein (Meardi 2006). Die aktuelle WSI-Betriebsrätebefragung 2007 bestätigt den Trend: Obwohl Outsourcing und Offshoring sowohl in kleinen als auch in großen Betrieben vorkommen, steigt mit der BetriebsgröBe die Wahrscheinlichkeit, dass Betriebe beide Umstrukturierungsmaßnahmen einsetzen. Abgerundet werden die Hinweise auf Größenabhängigkeit durch den signifikanten Einfluss von Haustarifverträgen auf Ausgliederungen. Haustarife sind in der Regel eher in Großbetrieben anzutreffen.

Standortverlagerungen werden in der Literatur vor allem mit dem produzierenden Gewerbe - als eine Arbeits-(kosten) intensive Branche - in Zusammenhang gebracht, während Outsourcing von Service- und Funktionsbereichen branchenübergreifend diskutiert wird. Unsere Schätzergebnisse zeigen beim Branchenvergleich eindeutige Unterschiede auf. Während sich Standortverlagerungen in der Grundstoffund Produktionsgüterindustrie, der Investitions- und Gebrauchsgüterindustrie sowie im Kredit- und Versicherungsgewerbe signifikant von der Referenzbranche private Dienstleistungen unterscheiden, gibt es mit Ausnahme des Wirtschaftsbereichs (privates) Kredit- und Versicherungsgewerbe bei Ausgliederungen keinen signifikanten Unterschied. Standortverlagerungen haben nach diesen Ergebnissen eine branchenbezogene Dimension: Bei Industriebetrieben wie auch bei Kredit- und Versicherungsunternehmen steigt das Chancenverhältnis signifikant an, Standortverlagerungen durchzuführen. Hingegen ist Ausgliederung eine Umstrukturierungsmaßnahme, die über die Branchen hinweg keinen signifikanten Effekt zeigt.

\section{5 \\ Betriebliche Folgen für die Beschäftigten}

Wenn die dominanten Beweggründe für Verlagerung und Ausgliederung reine Kostenaspekte waren, lässt sich vermuten, dass 
sich diese unmittelbar auf die Arbeitsplätze, auf die Beschäftigten und auf ihre Arbeitsbedingungen in den Betrieben ausgewirkt haben.

Es ist davon auszugehen, dass mit einer angekündigten Standortverlagerung oder Ausgliederung die Unsicherheit und die Angst um den eigenen Arbeitsplatz bei den Beschäftigten steigen, insbesondere dann, wenn das Kostenmotiv (Abschnitt 3) eine große Rolle spielt. Dies kann sich auch auf die Betriebsratsarbeit auswirken und die Beziehung zwischen Arbeitgeber und betrieblicher Interessenvertretung belasten. In der Tat wandeln sich nach der WSI-Betriebsrätebefragung die Inhalte der Betriebsratsarbeit massiv, sobald Standortverlagerung und/oder Ausgliederung in den Betrieben ein Thema wird. Wie die Schätzergebnisse zeigen (Tabelle 2), nahmen die betrieblichen Probleme rund um die Sicherung von Beschäftigung zu - bei Standortverlagerung noch deutlicher als bei Ausgliederung. Mit Themen wie Personalabbau, Sozialplänen, Einschränkung betrieblicher Sozialleistungen beschäftigten sich Betriebsräte in verlagerten Betrieben weitaus häufiger als betriebliche Interessenvertretungen in Betrieben, die keine Standortverlagerung durchführten. Für Betriebsräte in Ausgliederungsbetrieben steigt der tarifpolitische Regelungsbedarf zur betrieblichen Umstrukturierung in den Folgejahren an. Im Zuge von Offshoring und Outsourcing wurden zudem verstärkt betriebliche Bündnisse zur Sicherung der Beschäftigung geschlossen und/oder tarifliche Öffnungsklauseln genutzt. Betriebsräte machten in diesen Fällen Zugeständnisse bei den Arbeitsbedingungen (Löhne, Arbeitszeiten), im Gegenzug gab der Arbeitgeber häufig eine Standort- bzw. Beschäftigungsgarantie.

Zudem ist eine betriebliche Diskussion um die Auswirkungen von Standortverlagerung und Ausgliederung meist spannungsgeladen. Die Beschäftigten werfen dem Management oftmals mangelnde Loyalität und „Verrat" an den Beschäftigten vor, wenn es darum geht, Teile des Betriebs aus Gründen der Kostenoptimierung zu verlagern und dabei festes Stammpersonal im Betrieb zu entlassen. Der Betriebsrat sitzt dabei zwischen den Stühlen, er muss zwischen Arbeitgeber und Beschäftigten vermitteln und Arbeitsplätze im eigenen Betrieb weitestgehend erhalten. Oftmals - vor allem in Ausgliederungsbetrieben - wurden Mitwirkungsrechte des Betriebsrats behindert.

\section{Abb. 2: Arbeitsplatzeffekte von Standortverlagerung und Aus- \\ gliederung - Angaben der Betriebsräte* in \% (Mehrfachnennungen) -}

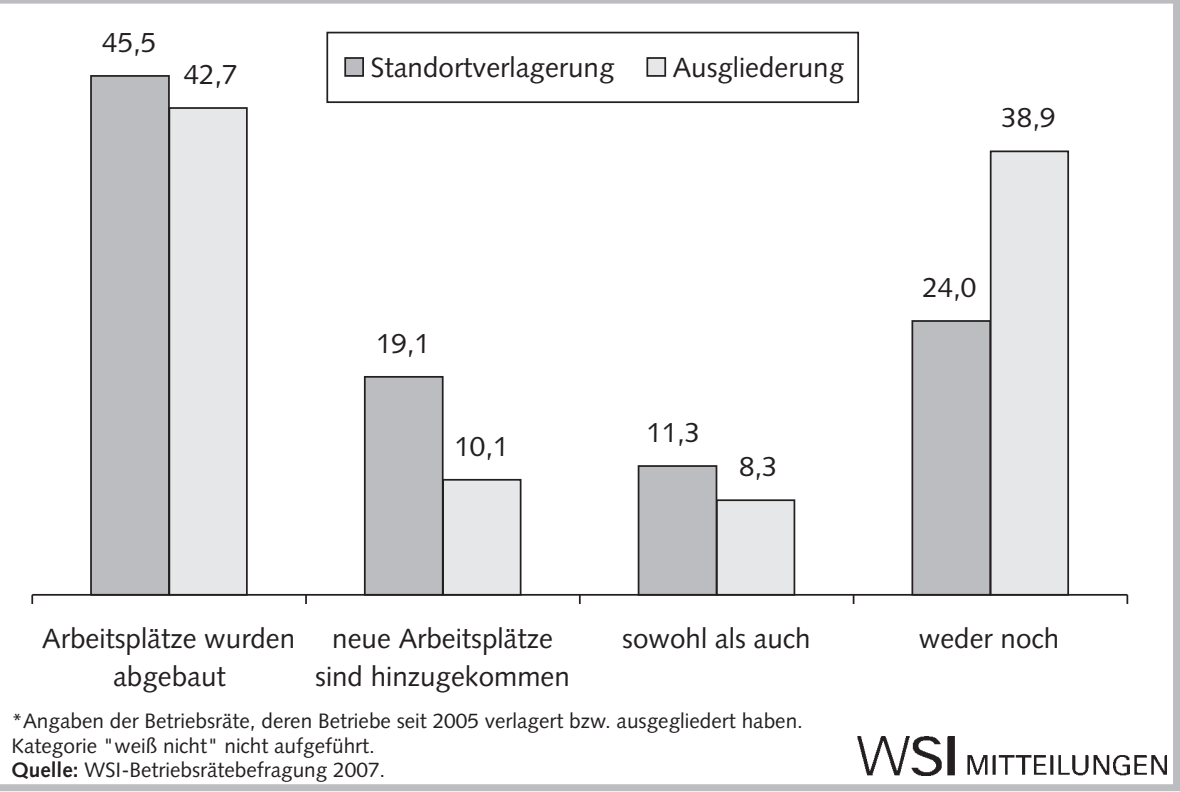

\subsection{ZUR ARBEITSPLATZENT- WICKLUNG}

Die Diskussion um die Auswirkungen von Standortverlagerungen und Ausgliederungen auf Beschäftigte und Arbeitsplätze wird in zwei Richtungen geführt: Einerseits werden vor dem Hintergrund des Kostenaspekts in Deutschland beide Umstrukturierungsmaßnahmen vor allem im Hinblick auf einen Arbeitsplatzabbau diskutiert. Der Arbeitsplatzverlust in Folge einer Standortverlagerung oder einer Ausgliederung ist für die betroffenen Beschäftigten die einschneidendste Auswirkung. Andererseits stellen insbesondere bei Verlagerungen Studien den Zusammenhang zur nationalen Wettbewerbsfähigkeit her. Danach müssen sich deutsche Unternehmen im Ausland engagieren, um ihre herausragende Stellung zu behaupten und neue Märkte zu erschließen. Ein Auslandsengagement sichere heimische Arbeitsplätze, wenn dadurch nicht sogar neue Arbeitsplätze entstünden - so die Argumentation. Nach Berechnungen der ILO (International Labour Organisation) entfiel in den 1990er Jahren auf einen Arbeitsplatz im Ausland durch ausländische Direktinvestitionen ein neuer Arbeitsplatz im Inland (Madeuf 1995). Auch für Deutschland unterstreichen aktuelle Studien die positive makroökonomische Wirkung von Auslandsinvestitionen. So geht das Institut für Weltwirtschaft (Klodt 2004) davon aus, dass in der Automobilindustrie auf drei im
Ausland entstandene neue Arbeitsplätze ein neuer Arbeitsplatz im Inland kommt. Trotz dieser Ergebnisse ist es aber für Deutschland in der Summe bisher nicht möglich, einen positiven oder negativen Arbeitsplatzsaldo aus den Verlagerungsoder Ausgliederungsaktivitäten abzuleiten.

Die makroökonomische Sicht dieser Studien liefert im konkreten Fall nur einen ungefähren Hinweis auf den Zusammenhang von Standortverlagerung, Ausgliederung und Beschäftigungsentwicklung. Die Betriebsräte wurden deshalb vom WSI auch danach gefragt, ob durch die vollzogene Standortverlagerung und Ausgliederung Arbeitsplätze abgebaut oder neue hinzugekommen sind. ${ }^{3}$ Zwar können durch die Befragungsergebnisse nicht jeder Verlagerung und jeder Ausgliederung exakte Arbeitsplatzeffekte zugewiesen werden. Sicher ist aber, dass Standortverlagerung und Ausgliederung - den Befunden der WSI-Befragung zufolge - oftmals vor dem Hintergrund eines gleichzeitig ablaufenden Personalabbaus stattfanden, bei Standortverlagerung etwas häufiger als bei Ausgliederungen (Abbildung 2). Dieses Ergebnis deckt sich mit den oben stehenden

\footnotetext{
3 Die Angaben der Betriebsräte beziehen sich auf ihren Betrieb, weiterführende Arbeitsplatzeffekte, die im Zusammenhang mit der Verlagerung oder Ausgliederung in anderen Betrieben entstanden sind, werden über die Befragungsergebnisse nicht erfasst.
} 


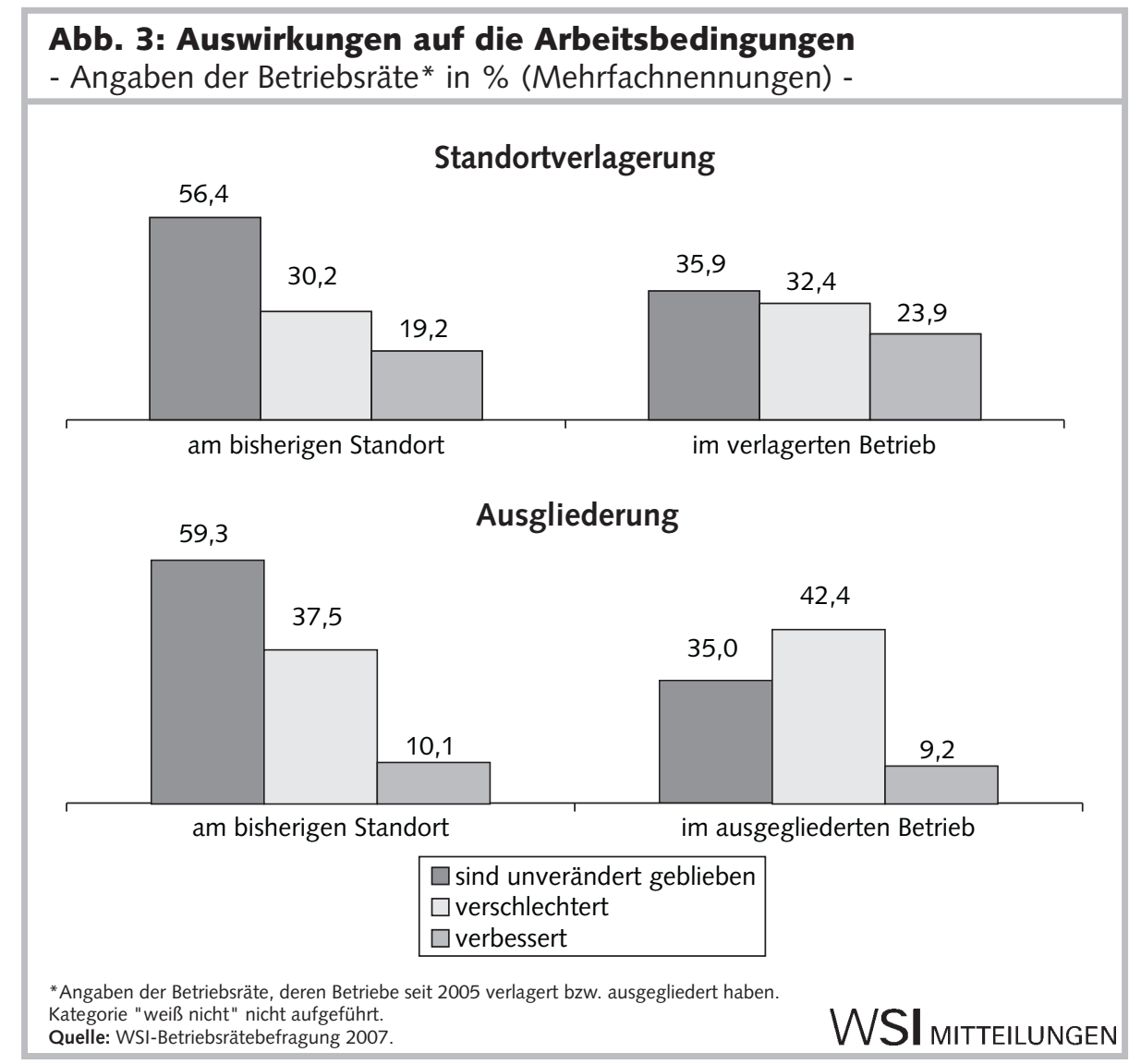

Ausführungen, dass kostengetriebene Unternehmensverlagerungen und -ausgliederungen negative Auswirkungen auf die Arbeitsplätze haben. Nicht selten (in fast jedem fünften verlagernden Betrieb) entstanden aber auch neue Arbeitsplätze. In jedem vierten verlagernden Betrieb und in mehr als jedem dritten ausgliedernden Betrieb kam es weder zum Abbau noch zum Aufbau von Arbeitsplätzen. Unklar bleibt, in welchem quantitativen Ausmaß der Arbeitsplatzabbau und -aufbau geschah, denn konkrete Zahlen im Hinblick auf Entlassungen und Einstellungen gibt die WSIBefragung nicht her.

Wie andere Studien zum Arbeitsplatzabbau bei Standortverlagerungen zeigen, sind vor allem Menschen mit niedrigerem Qualifikationsniveau davon betroffen (Eurofound 2006). Da von der Verlagerung besonders stark die personalintensiven Bereiche und von der Ausgliederung Servicefunktionen tangiert waren, stieg der Wettbewerb um die verbliebenen Stellen. Die bisher als sicher geltenden Beschäftigungsverhältnisse wurden infolge der Verlagerungen und Ausgliederungen zunehmend prekär und unsicher. Dadurch stieg auch der Druck auf die Arbeitsbedingungen aller Beschäftigten an.

\subsection{AUSWIRKUNGEN AUF DIE ARBEITSBEDINGUNGEN}

Aber auch wenn der einzelne Arbeitsplatz in seiner Existenz nicht gefährdet ist, können Standortverlagerungen und Ausgliederungen massive Auswirkungen in den Betrieben haben. Arbeitgeber nutzen die Verlagerung oder die Ausgliederung, um sowohl am bisherigen Standort als auch im verlagerten bzw. ausgegliederten Betrieb Druck auf die Beschäftigten auszuüben und z. B. die Löhne zu senken oder Arbeitszeiten auszuweiten.

$\mathrm{Ob}$ und wie sich die Arbeitsbedingungen in den Betrieben nach einer Standortverlagerung und Ausgliederung veränderten, hält Abbildung 3 fest. Verlagerungen und Ausgliederungen hatten am bisherigen Standort in gut der Hälfte der Betriebe keine Auswirkungen auf die Arbeitsbedingungen. Fast ein Viertel der Betriebsräte mit Standortverlagerungen und $10 \%$ mit Ausgliederungen schätzten die Arbeitsbedingungen sogar besser ein als vor der Maßnahme. Dies machten sie daran fest, dass nach der durchgeführten Maßnahme die Arbeitsplätze sicherer geworden sind und die Arbeitsorganisation optimiert wurde. Trotzdem gab es einen nicht zu ver- nachlässigenden Anteil an Betrieben, in denen sich die Arbeitsbedingungen an den bisherigen Standorten verschlechtert hatten. Dies traf für 37,5\% der Betriebe mit Ausgliederungen und 30,2 \% mit Standortverlagerungen zu. In der Mehrzahl der Betriebe mit schlechteren Arbeitsbedingungen geschah dies, weil für die Beschäftigten der Arbeitsdruck angestiegen ist, das Betriebsklima sich verschlechtert hatte und die Beschäftigten nach der Umstrukturierungsmaßnahme mehr und länger arbeiten mussten als vorher. Zusätzlich kam es zwischen den Standorten zu Kommunikationsproblemen. Die Betriebsräte der verlagernden bzw. ausgliedernden Betriebe vertraten die Ansicht, dass trotz der Umstrukturierung die verbliebenen Arbeitsplätze unsicherer geworden sind. Dieses Bild findet man auch in den verlagerten Betrieben vor, allerdings in etwas anderen Größenordnungen.

Am negativsten stellten sich allerdings die Auswirkungen auf die Arbeitsbedingungen in den ausgegliederten Betrieben dar. 42,4\% der Betriebsräte aus den ehemaligen Betrieben ${ }^{4}$ gaben an, dass sich dort die Arbeitsbedingungen für die Beschäftigten verschlechterten, wohingegen $35 \%$ von gleich gebliebenen Arbeitsbedingungen berichteten und noch nicht einmal $10 \%$ von besseren.

\section{Fazit}

Standortverlagerungen und Ausgliederungen sind kein vorübergehendes Phänomen. Beide haben sich als Managementinstrumente etabliert, die insbesondere nach Aussagen der befragten Betriebsräte zur Kostenoptimierung eingesetzt werden. Beide Umstrukturierungsmaßnahmen beeinflussen die Arbeitswelt nachhaltig.

Nach den Erfahrungen, die Betriebsräte mit Standortverlagerungen und Ausgliederungen gemacht haben, sehen sie die beiden Maßnahmen differenziert (Tabelle 3). Bei Betriebsräten mit Standortverlagerungserfahrungen zeigt sich im Antwort-

4 Die Mehrzahl der Betriebsräte aus den ehemaligen Betrieben ( $85 \%$ ) hatten Informationen über die Arbeitsbedingungen in den entstandenen, ausgegliederten Betrieben. 
verhalten auf die Frage, wie sie im Nachhinein die Standortverlagerung beurteilten, eine relativ große Ambivalenz. Die Hälfte von ihnen beurteilte die angekündigte Standortverlagerung zunächst positiv. Nach der durchgeführten Verlagerung sagten sogar 58 $\%$ der Betriebsräte aus, dass die Standortverlagerung Sinn machte. Aber sie hat andererseits dem Betrieb neue Probleme gebracht und die Arbeitsplätze wurden dadurch nicht immer sicherer. Anders sieht die Einschätzung zu Ausgliederungen aus. Von vornherein stand die Mehrzahl der Betriebsräte (nämlich $70 \%$ ) den Ankündigungen von Ausgliederungen negativ gegenüber. Auch nach vollzogener Maßnahme änderten sich die Einstellungen der Betriebsräte nicht. Nur für etwas mehr als jeden dritten Betriebsrat machte die Ausgliederung im Nachhinein Sinn. Und auch bei Ausgliederungen wurden die Arbeitsplätze im Unternehmen nicht sicherer und die betrieblichen Probleme, wie der Druck auf die Arbeitsplätze, stiegen an, obwohl bei Ausgliederung wie auch bei Verlagerung fast die Hälfte der Betriebsräte aussagte, dass das Unternehmen nachher wirtschaftlich besser gestellt war als vor der Maßnahme.

\begin{tabular}{|c|c|}
\hline \multicolumn{2}{|l|}{ Standortverlagerung } \\
\hline Die Standortverlagerung machte Sinn & 58,0 \\
\hline Die Standortverlagerung hat die Arbeitsplätze im Unternehmen sicherer gemacht & 42,7 \\
\hline Das Unternehmen steht heute wirtschaftlich besser da als vor der Verlagerung & 43,5 \\
\hline $\begin{array}{l}\text { Durch die Standortverlagerung ist weiterer Druck auf die Arbeitsplätze und die } \\
\text { Beschäftigten ausgeübt worden }\end{array}$ & 51,4 \\
\hline Die Standortverlagerung hat innerbetrieblich neue Probleme erzeugt & 64,4 \\
\hline \multicolumn{2}{|l|}{ Ausgliederung } \\
\hline Die Ausgliederung machte Sinn & 36,2 \\
\hline Die Ausgliederung hat die Arbeitsplätze im Unternehmen sicherer gemacht & 23,7 \\
\hline Das Unternehmen steht heute wirtschaftlich besser da als vor der Ausgliederung & 42,1 \\
\hline $\begin{array}{l}\text { Durch die Ausgliederung ist weiterer Druck auf die Arbeitsplätze und die } \\
\text { Beschäftigten ausgeübt worden }\end{array}$ & 59,2 \\
\hline Die Ausgliederung hat innerbetrieblich neue Probleme erzeugt & 70,4 \\
\hline Quelle: WSI-Betriebsrätebefragung 2007. & UNGEN \\
\hline
\end{tabular}

Mit der Verbreitung der beiden Umstrukturierungsmaßnahmen Ausgliederung und Standortverlagerung ist in den Betrieben die Einsicht gewachsen, dass Geschäftsführung und betriebliche Interessenvertretung ein geordnetes Verfahren brauchen, um den Umgang mit Umstrukturierungsmaßnahmen zu regeln und das Konfliktpotenzial im Betrieb zu minimieren. Deshalb werden mittlerweile Betriebsvereinbarungen $\mathrm{zu}$ Umstrukturierungsmaßnahmen abgeschlossen (Müller/Mül- ler 2000). Dabei gehört die Abfederung von sozialen Folgen von Fremdvergaben zu den klassischen Regelungsinhalten der Betriebsparteien. In nächster Zukunft sehen die Betriebsräte an diesem Punkt weiteren Handlungsbedarf. Nach der WSI-Betriebsrätebefragung 2007 würden sie es sogar begrüßen, wenn auf übergeordneter Ebene in den nächsten Jahren tarifpolitische Regelungen betriebliche Umstrukturierungen absichern würden.

\section{LITERATUR}

Ahlers, E./Öz, F./Ziegler, A. (2007): Standortverlagerung in Deutschland - einige empirische und politische Befunde, edition der Hans-Böckler-Stiftung 194, Düsseldorf

Buch, C./Schnitzer, M. (2007): Analyse der Beweggründe, der Ursachen und der Auswirkungen des sogenannten Offshoring auf Arbeitsplätze und Wirtschaftsstruktur in Deutschland, Tübingen und München Deutscher Industrie- und Handelskammertag (DIHK) (2005): Investitionen im Ausland. Ergebnisse einer DIHK-Umfrage bei den Industrie- und Handelskammern, Berlin

Dressler, S. (2007): Off-Shore - Die Erosion von Arbeitsplätzen?, in: Fischer, J./Nullmeier, E. (Hrsg.): Hauptsache Arbeit?, Frankfurt a. M. u. a., S. 53-74

\section{European Foundation for the Improvement of Living and Working} Conditions (2006): Restructuring and employment in the EU: Concepts, measurements and evidence, Dublin

Görzig, B./Kaminiarz, A./Stephan, A. (2005): Wie wirkt sich Outsourcing auf den Unternehmenserfolg aus? Neue Evidenz, in: Schmollers Jahrbuch 125, S. 489-507

Haipeter, T./Banyuls, J. (2007): Arbeit in der Defensive? Globalisierung und die Beziehungen zwischen Arbeit und Kapitel in der Automobilindustrie, in: Leviathan 3, S. 373-400
Hofmann, I./Leitsmüller, H./Samsinger, R. (2002): Umstrukturierung Fusion, Outsourcing, Ausgliederung Leitfaden für Betriebsräte, Wien Kinkel, S./Spomenka, M. (2008): Produktionsverlagerungen rückläufig. Ausmaß und Motive von Produktionsverlagerungen und Rückverlagerungen im deutschen Verarbeitenden Gewerbe, Mitteilungen aus der ISI-Erhebung zur Modernisierung der Produktion 45, Karlsruhe

Kinkel, S./Lay, G./Maloca, S. (2004): Produktionsverlagerungen ins Ausland und Rückverlagerungen, Forschungsbericht, Fraunhofer-Institut für Systemtechnik und Innovationsforschung, Karlsruhe

Klodt, H. (2004): Mehr Arbeitsplätze durch Auslandsinvestitionen, in: Die Weltwirtschaft 4, S. 373-398

Madeuf, B. (1995): Foreign Direct Investment, Trade and Employment Delocalisation, in: OECD (Hrsg.): Foreign Direct Investment, Trade and Employment, Paris, S. 41-65

Meardi G. (2006): The Complexity of Relocation and the Diversity of Union Responses: Efficiency-oriented FDI in central Europe, Vortrag gehalten auf dem FAFO-FES-Workshop am 10-11.11. in Oslo Müller, S. G./Müller, M. (2000): Outsourcing. Analyse und Handlungsempfehlungen, edition der Hans-Böckler-Stiftung 37, Düsseldorf 